\title{
XXX. On the methods employed for determining the ohm
}

\section{G. Wiedemann}

To cite this article: $G$. Wiedemann (1882) XXX. On the methods employed for determining the ohm , Philosophical Magazine Series 5, 14:88, 258-276, DOI: 10.1080/14786448208627264

To link to this article: http://dx.doi.org/10.1080/14786448208627264

Published online: 08 Jun 2010.

Submit your article to this journal ¿

Џll Article views: 2

Q View related articles $\square$ 
lead by the employment of more powerful field-magnets. In all cases the lead must be ascertained by its being the position of least sparking, and the brushes set pretty closely to it; otherwise the brushes are burned away, and the sparking is unpleasant.

Having charged the accumulators, I divided them up into circuits as required, and employ them to work my electropneumatic and other appliances. I have driven every thing for a week after a charge of a couple of hours; but on account of the local action in the red-lead cells, this is not a very advantageous course; and latterly I have preferred to charge for half an hour in the morning of each day. If the charge is meant to last, of course the currents must be used economically.

There are considerations, however, connected with the inevitable variation in the power of the current when any description of cells is used as a source of electricity, which make me already doubtful whether this alternate procedure will answer, and whether it may not be necessary to use the cells with the machine running simply for the purpose of sluicing off small currents with fairly constant differences of tension at their sources from the main current of the machine. It is even possible that simple pairs of lead plates in acid withont preparation may be sufficient for this purpose. But the discussion of this matter must wait until my uniform rotation-machines are in a more complete state.

I will close with an experiment showing, in a manner suitable for the lecture-table, the development of a counter electromotive force in the dynamo when driven by a current from the accumulators.

I place a Swan lamp in parallel circuit with the machine. Then, so long as the machine is at rest, the lamp does not burn. But when the machine is in motion, as the velocity increases the counter electromotive force is set up, and the passage of the current through the machine more or less completely barred. The lamp in the parallel circuit then burns up. If the machine is stopped by a brake or overloading, the lamp is extinguished again.

XXX. On the Methods employed for determining the Ohm. By G. WIEDEMANN*.

$7 \mathrm{THE}$ Congress of Electricians which met at Paris in the 1 autumn of last year adopted the electromagnetic units based on the centimetre-gramme-second system for the con-

* Translated from the Elektrotechnische Zeitschrift, July 1882. From a separate impression, communicated by the Author. 
stants of the galvanic current, as fundamental units, and expressed a wish that a special international commission should be intrusted, in the first place, with the construction of a standard ohm as unit of resistance. Since, then, further consultation is to take place before very long, it seems desirable to consider again the methods hitherto employed, with their sources of error, from the experimental standpoint; their mathematical theory has been sufficiently discussed.

I desire also to give an impulse to further discussion of the methods to be adopted in the new determination. This is to be done the more strictly and thoroughly, since the units, having been once determined, ought not to be altered again immediately in consequence of further investigations.

It is well known that $W$. Weber, to whom we owe the fundamental facts in this subject, has given four methods of obtaining a conductor of given resistance in electromagnetic units:-

I. A circle of wire of known dimensions is caused to revolve through a certain angle about an axis (vertical) inclined to the direction of the earth's magnetism; and the intensity of the current thus induced is measured by means of a galvanometer of known dimensions. The intensity of the current, under similar conditions, is inversely proportional to the resistance of the conductor.

II. Instead of measuring the dimensions of the galvanometer in the first method, the action of the unit current on the magnetic needle of a multiplier is determined by the damping of its oscillations when the circuit is closed.

III. A magnetic needle is allowed to oscillate within a closed multiplier of known dimensions, and the damping of its oscillations is determined.

IV. A circle of wire is put into uniform rotation about a horizontal or vertical diameter; and we observe the deflection which takes place in a magnetic needle swinging at the centre of the circle, in consequence of the current induced in the revolving circle by the earth's magnetism. Since in carrying out these methods each separate measurement is of necessity attended with error, that method appears at the outset the most reliable which involves the determination of the fewest constants, and in which these determinations can be made with most accuracy. Hence methods III. and IV. appear at the outset to offer special advantages.

We will consider the fourth method first, in order to discuss at once a number of sources of error which partly affect also the other methods. This method is in fact that employed 
by the Committee of the British Association appointed for the purpose in 1863.

A wire coil is put into rotation about a vertical axis, in consequence of which currents are induced in it, whose intensity in unit time is, on the one hand, directly proportional to the horizontal component of the earth's magnetism and to the change of the projection of the plane of the coil on the vertical plane at right angles to the direction of that component, and, on the other hand, inversely proportional to the resistance of the coil.

But, then, the induced currents which traverse the coils act upon the neighbouring coils, and induce in them extra currents whose electromotive force is proportional to the change in the directly induced current, in the unit time; hence the inductive action in the spiral is diminished. The deflection of the needle is determined by the total action of all the induced currents. The following investigations are therefore necessary to determine the absolute resistance of the coil.

1. The measurement of the space enclosed by the windings of the coil, to which the induction is, cateris paribus, proportional, as well as its form, upon which the extra currents induced in it depend, and the moment of rotation exerted on the magnetic needle at its centre.

These determinations offer very considerable difficulty.

If thick wire is employed for winding the coil, its diameter, together with the insulating covering, must be exactly an aliquot part of the interior width of the frame on which the wire is to be wound, or else the outer layers of wire will be squeezed more or less between the wires of the inner layers, and so cause displacement. Moreover, as W. Siemens* has shown, the wire is stretched in winding, the more the thinner the wire is; and this extension may be as much as 6 per cent. Again, the insulating covering of the wire becomes pressed together; this takes place less when the corering consists of solid gutta-percha or similar substance than when the wire is covered with silk or cotton. The thinner the wire is, the more important do these errors become, of displacement of the wire, of extension on winding, and of the squeezing together of more of the insulating covering in proportion to the diameter of the wire. It is therefore not correct to calculate the space enclosed by the coils from the length of the wire before winding or after unwinding, and from the dimensions of the coil.

On this account, as W. Siemens rightly remarks, the accurate measurement of the length of the wire of about 1.1 milli-

* Poggendorff's Annalen, 1866, vol. cxxvii. p. 327. 
metre thickness to the tenth of a millimetre, as in the older experiments of the British Association, was useless.

It is not difficult to determine the internal diameter, in various directions, of the coils of wire (that is, the diameter of the frame on which they are wound), either by means of a kathetometer, or with an inextensible steel tape of constant temperature; but, on the other hand, the measurement of the external diameter or circumference is much more difficult, in consequence of the inequality of the covering of the wire and the unevenness of the surface.

If the error in the determination of the mean diameter only amounted to 0.5 millim., which, in view of the circumstances mentioned, is certainly not an extreme estimate, then in the case of the coil of 314 millim. diameter employed by the Committee of the British Association the estimate of the space enclosed by the coils would be wrong by $\frac{1}{314}=0.32$ per cent. In order to reduce this error as much as possible, it is necessary, as both W. Weber * and Lord Rayleigh† recommended, to take the diameter of the coil as great as possible consistently with accuracy of rotation. Also the wire should be wound with a tension as uniform as possible; and the exterior diameter should be controlled after each layer has been wound.

A much more important source of error lies in the uncertainty of determining the mutual position of the separate coils, depending upon the conditions explained above, upon which the intensity of the extra current induced in the coil when put into rotation depends. Since the inductive action of the coils upon each other takes place at very small distances, a very small error in measuring the distance apart is of great importance.

A further source of inaccuracy is introduced by making the coil of two parts parallel to each other, but with a space between in order to admit the thread by which the magnet is suspended; so that here also the parallelism and distance apart of the two portions must be very exactly determined. That the data may be greatly altered by the extra currents is seen from the fact that, in an experiment of a Committee of the British Association, the position of maximum induction of the coil in rapid rotation was displaced by not less than $20^{\circ}$; and the correction for this amounted to some 8 per cent.

If we seek to determine the self-induction by opposing the coil to another of known coefficient as a Wheatstone's bridge $\ddagger$,

* Berichte der Königlich Sächsischen Gresellschaft der Wissenschaften, 1880, p. 77.

$\dagger$ Proceedings of the Royal Society, 1881, vol. xxxii. p. 122.

† Compare Maxwell's 'Treatise,' vol. ii. p. 357; Brillouin, Comptes Rendus, vol. xciii. p. 1010 (1881); Beiblatter, vol. vi. p. 39. 
then we have, besides the sources of error of the original apparatus, a number of other sources of error which need to be specially examined; so that it is possible the accuracy of the results might be seriously prejudiced. In any case the difficulty of accurately determining the self-induction is the most suspicious part of the method under consideration.

2. The temperature of the coil must be determined with the greatest accuracy, since the conductivity of the wire decreases about 0.3 per cent. for a rise of temperature of $1^{\circ} \mathrm{C}$. The corresponding change of length, and consequently of surface embraced, amounts only to 1000000 , and may therefore be neglected.

3. We have further to inquire whether there may not be secondary currents induced in the supports of the apparatus, if these are of metal, by the currents circulating in the spiral, which may act upon the magnetic needle*.

This point may be determined by interrupting the continuity of the metallic supports by means of insulating material. According to experiments of this nature, made by Lord Rayleigh and Dr. Schuster $\dagger$, this source of error was not important in the experiments of the British Association, the error amounting only to $0 \cdot 16$ per cent. It would be better, however, to construct the supports of insulating material.

4. The testing of the instrument to determine if the coils lie symmetrically with reference to the axis of rotation offers no particular difficulty, if we observe by means of a telescope corresponding points of the frame on both sides of the axis in different positions of the coils, making with each other an angle of $180^{\circ}$.

5 . In the same way it is easy to ascertain by known optical methods whether the axis of rotation is really vertical, and remains so; deviation from the vertical position may exert a considerable effect.

If the angle of dip were about $\angle=70^{\circ}$, an inclination of the axis towards north or south of $0^{\circ} .2$ would cause an alteration in the inductive action of the earth in the proportion of $\cos 70^{\circ}: \cos \left(70^{\circ} \pm 0^{\circ} \cdot 2\right)$ 一 that is, not less than 1 per cent. An inclination of this amount in the case of the British-Association coil would correspond to a displacement of 0.5 millim. in the ends of the axis. An exact determination is therefore very necessary. Small displacements of the axis towards east or west have only an insignificant influenco.

6. The counting of the number of revolutions of the coil in p. 9.

* Compare F. Kohlrausch, in Pogg. Ann. 1874, Ergänzungsband vi.

+ Loc. cit. 
unit time by known methods offers no special difficulty; nor does the maintaining of a constant velocity of rotation by mechanical means*.

7. The adjustment of the magnet in the centre of the rotating coil should also not be difficult to effect. Moreover a small deviation from exact adjustment causes no important error.

8. The moment of the magnet may be determined by vibration-experiments after determining its moment of inertia and the horizonal component of the earth's magnetism; or it may be determined from experiments on deflection. If, in order to render the inductive action of the magnet on the rotating coil imperceptible, we employ magnets of very small moment, then these methods offer many sources of error, on account of the very perceptible influence of the friction of the air in the vibration-experiments, or of the small distance at which the deflecting magnet must be placed.

If the moment of the magnet is very small, it and the distribution of magnetism in the magnet (which is very difficult to determine) have both very small effect upon the results. If the poles of the magnet are at a distance from the central point in the median plane of the coils which is less than $\frac{1}{6}$ of their radius, and if the distance of the poles from the plane is not greater than 0.84 of the length of the magnet, then the force exerted upon it by a current in the coil is constant to within 0.0005 , under conditions otherwise similar, up to a deflection of $56^{\circ}$.

9. The adjustment of the telescope and scale required for reading-off the position of the magnet, and the correction of the readings, may be made in the usual way; and the divisions of the scale must be compared with an accurate measure. The accurate measurement of the distance of the scale from the central point about which rotation of the magnet takes place, or from the reflecting surface of the mirror, offers a certain amount of difficulty.

10. The force of torsion of the suspension-thread of the magnet may easily be compared with the directive force of the magnet by turning the thread fastened to a torsion-circlo through a certain angle, and observing the deviation of the magnet. Unavoidable changes of considerable magnitude may result from the variable moisture of the air, if weak magnets are employed.

11. We have, further, to inquire what influence currents of air and the vibration of the apparatus caused by the motion

* Compare the ingenious arrangement adopted by Lord Rayleigh, loc. cit. 
exert upon the needle when the coil is put into rotation with circuit open. In the first experiments of the Committee of the British Association, in which attention had not yet been paid to the separate conditions of the experiment in the way which will be necessary for a final determination of the ohm, these last-mentioned sources of error made themselves in a high degree perceptible.

Thus F. Kohlrausch, in a discussion of these experiments, has justly pointed out that, in order to avoid inductive action on the coil of wire, the magnet (a magnetized steel ball of 8 millim. diameter), in spite of its great mass, had only a moment equal to that possessed by an extremely fine magnetic needle weighing 0.025 gramme. The magnet was attached by means of a wire 0.25 metre long to a mirror of 30 millim. diameter suspended by a simple silk fibre of 2 metres length. The currents of air produced by the rotating coil only 0.31 metre distant acting upon the relatively large surface of the mirror and magnet, as well as the variable torsion of the thread, become much too considerable in comparison with the directive force of the magnet.

Further, the vibration of the apparatus produced by the rotation may propagate itself to the case surrounding the mirror, and so put the air, and with it the mirror, into rotation. It might thus happen that the deflections obtained by rotating the coil in one or the other direction might vary by as much as 8.5 per cent. If the mean results obtained in different series of observations should differ amongst each other by only 2.3 per cent., yet even this is not a guarantee of greater accuracy, but can only be regarded as a proof that the apparatus always acts in nearly the same way.

Moreover the supplementary elimination of sources of error, e. $g$. by more exactly calculating the effect of self-induction, as attempted in the memoir of Lord Rayleigh and Dr. Schuster, can in no way free the results from the influence of sources of error shown to exist by the deviations cited above. Above all things, observations of this kind ought never to be arbitrarily corrected on the ground of probability only without having perfectly definite numerical data; or else all secure experimental ground is lost.

Hence we may consider it shown that the results of these experiments are not to be themselves taken as a final determination of the ohm, but rather as extremely valuable preliminary experiments by which we have become acquainted with the precautions to be observed.

By means of new experiments made by Lord Rayleigh and Dr. Schuster with the apparatus of the British Association, 
altered in some points, the difficulties mentioned above under (1) remained unaltered; the magnet, however, was replaced by four magnetized needles, each 0.5 centim. long, fastened on the parallel horizontal edges of a cube of cork. The mirror was attached directly to the cork. But here also the directive force of the magnet is small in comparison with the somewhat powerful influence of currents of air acting on the large surface and small moment of inertia.

Further, the case protecting the suspended portions of the apparatus is attached to the glass tube which surrounds the suspension-thread. Perfect stability could hardly be obtained with such an arrangement; since with rapid rotation vibration of the mirror would certainly be experienced. These experiments also are to be regarded more as preparatory for later and final measurements, and as such have been excellently carried out. On this account no doubt the more exact data for ver. tical adjustment of the axis \&c. have not yet been given throughout. Lord Rayleigh, as already mentioned, lays emphasis on the necessity for new coils for the final experiments, and raises the question whether these should not be arranged as in the Helmholtz-Gaugain galvanometer, so that the directive action of the current on the needle is independent of any small excentricity or deviation of the needle.

In the final experiments, whether they are made according to this method or according to one of the other methods, it is in any case necessary that as complete a statement should be given of each separate proceeding in arranging and using the apparatus. Also the experiments should not be made with an apparatus set up and adjusted once for all, since then the errors of the arrangement will repeat themselves with each new determination undertaken with the apparatus. On the contrary, the apparatus itself must be frequently altered in various ways. Only so can we obtain results independent of each other, which can be used for mutual control.

The sources of error which are so difficult to avoid in using method IV., more particularly in consequence of the extra currents, have induced W. Weber* himself, in conjunction with the late F. Zöllner, to again take up the first method (which had been employed by the first-mentioned so long ago as 1846 in preliminary experiments) with the most perfect experimental means. Of two equal coils, weighing some $207 \mathrm{kilog}$., most'carefully and very regularly wound upon mahogany rings, as shown by measurement of different diameters, the

* W. Weber and F. Zöllner, loc, cit. 
coils consisting of cotton-covered copper wire about $3 \frac{1}{3}$ millim. in thickness, and having a resistance of about 5 ohms-one, the multiplier, surrounds a cylindrical magnetic needle about 100 millim. long and 10 millim. thick, fxed in the magnetic east and west plane. The diameter of the coils (interior about 960 millim., exterior 1040 millim., and breadth of coils about 254 millim.) is so large that the magnetic forces acting on the needle may be supposed, without perceptible error, to be concentrated at their centre. The other coil, connected with the first by conducting-wires, the inductor, is capable of rotation about a vertical axis, out of the east and west position through an angle of $180^{\circ}$. This displacement of the inductor takes place suddenly at such times that the currents induced in it and transmitted through the multiplier render the deflections of the needle constant according to the "method of multiplication " or the " method of recoil."

In carrying out the experiments, the displacement of the inductor by means of clockwork was not found to be practicable, and it was therefore effected by hand. The time required for the displacement (some 2 seconds) must only be a small fraction of the time of vibration of the magnet hanging in the multiplier. For this purpose the time of vibration of the needle is increased (up to 30 seconds) by placing the needle in a stirrup to which a tube of brass 272 millim. long was fastened in a horizontal position and at right angles to the needle, the ends of which carried plane mirrors. Telescopes with parallel scales are placed before both mirrors, each at a distance of 4 millim. By this double reading the difficult measurement of the scales from the mirrors is replaced by the easier measurement of the distance of the scales from each other, and of the distance of the mirrors apart. An examination of the point whether, and how far, the instants of displacement of the inducing coil may differ from those indicated by calculation, and how far these possible deviations may affect, might be easily made*. In any case, according to the experiments which have been made, this infuence cannot be of importance, since the results obtained at different times by use of a magnet 100 millim. long agree with each other to within 0.06 per cent., and those obtained with a magnet 200 millim. long do not differ at all from the mean result. This shows at the same time that the instrument had not altered in any important respect in the interval. The inductor must be so far removed from the multiplier that the currents in the first cannot directly deflect the needle.

* Compare Chwolson, Bulletin de St. Pétersbourg, vol. ii. p. 403 ; Beiblätter, vol. v. p. 450 . 
It is a point of very great importance in the employment of this method that self-induction in the inductor is without influence, and that, further, the intensity of the earth's magnetism does not enter into the calculation, provided that it is of equal intensity in the positions occupied by inductor and multiplier. It may not be allowable to assume that this is the case in ordinary rooms, because of iron hooks let into the walls; but in a building constructed for the purpose this condition might easily be fulfilled. This point may also easily bo controlled by oscillation-experiments; the ratio of the directive force of the earth may be determined at the two places.

It is true that we have to set against this advantage that we have two coils to measure, and are so liable to a double error. (This, howevor, is also the case in the experiments of the British Association described above, where the revolving coil consists of two separated halves.) This disadvantage may be considered small in comparison with the error resulting in the fourth method from the measurement of the self-induction of the coil. It is further reduced by the large dimensions of the coils. At the same time stability of the apparatus, and consequent freedom from disturbances resulting from vibration and from currents of air, is much more easily obtained than with the rapidly-rotating coil employed in the former method.

Previous communications on experiments with this apparatus are only preliminary, on which account we are not yet in possession of details of adjustment; for which, however, not only the name of W. Weber, but also that of Repsold, the instrument-maker intrusted with the work, give us full guarantee that such points as the verticality of the axis of rotation, the adjustment of the coil magnetic east and west, the arrangement for displacing the coil through exactly $180^{\circ}$, and so on, will be attended to. The preliminary method of measuring the circumference of the mahogany rings on which the coils are wound and of their exterior circumference by means of strips of paper, which were then compared with a divided wooden rule, will no doubt give place to more exact methods in the actual experiments.

A special advantage of this method is that the wires may be unwound from the rings and wound again without any great difficulty; and so repeated observations independent of each other can be obtained.

This method is therefore to be recommended for the final determinations.

If we employ Weber's second method, we have no need to determine the dimensions of the multiplier; but we determine 
the damping of the oscillations of the needle, once with open circuit and again with closed circuit. Hence, by determining the deviation of the needle when the inductor is displaced, the resistance of the circuit in electromagnetic measure may be calculated. For this purpose the multiplier must enclose the needle more closely, so that the damping may be sufficient. We have to determine, besides the dimensions of the inductor, the period of oscillation and moment of inertia of the needle and the intensity of the horizontal component of the earth's magnetism. The sources of error of this method have been carefully examined by F. Kohlrausch*; the error in measurement of the inductor may, however, have been scarcely estimated high enough. (Compare also the following method.)

If proper experimental means and a suitable observatory are available $\dagger$ for accurate determination of the earth's magnetism, this method may very well be employed together with Weber's first method.

In Weber's third method, which is apparently so simple, we have, in order to determine the absolute resistance of a multiplier, to observe only the damping of the oscillations of a needle suspended in the coil when the ends of the coil are united, and again when the circuit is open. In order to be able to calculate the electromotive force due to the oscillations of the needle and its action upon the needle, which is directly proportional to that electromotive force, and inversely proportional to the resistance to be measured, we must know in general the dimensions of the multiplier and the position of the needle relative to it, as well as the distribution of the magnetism in the needle.

The former determinations are difficult to carry out exactly, since, in order that there may be sufficient damping, no very large dimensions can be given to the coil ; the latter determinations can only be made inexactly by observation of the currents induced in a short coil at different points of the magnet, or by numerous oscillation-and deflection-experiments with a magnetic needle swinging at different distances from various points of the magnet. We have also to satisfy ourselves that the damping is not dependent upon the angle of deflection of the needle.

This method has been employed by Fr. Weber $\ddagger$, in Zürich,

* Lac. eit.

† Compare also the new methods by F. Kohlrausch, Göttinger Nachrichten, March 4, 1882.

† F. Weber, Elektromagnetische und kalorimetrische Messungen (Zürcher und Furrer, Zürich, 1878); Beiblätter, vol. ii. p. 499. 
employing a magnet of 80 millim. length, $20 \cdot 1$ millim. breadth, and $21 \cdot 1$ millim. depth, suspended by a silk thread between two conical coils very regularly wound, of 144.43 millim. internal, 184.46 millim. external radius, and 51.64 millim. breadth.

The mean distance of the spirals from each other was either very small, or the distance of their median planes was made 164.4 millim., or very nearly equal to the mean radius of the coils. In this last case the distance of the poles from each other has no important influence, although it has in the first, where also the distribution of moments in the magnet must not be neglected. We must, however, always take into account inevitable differences in measurement in consequence of the mutual pressure of the coils and certain unavoidable irregularity in the winding, which are more important in consequence of the small dimensions of the coils, as in the fourth method which we have already considered. Nevertheless it was shown, by comparison with a standard resistance of Siemens's, with which the resistanee of the coils was compared by means of a Wheatstone's bridge, in both the cases mentioned, and after rewinding of the coils, that the standard resistance expressed in absolute measure varied in three series of experiments only from 0.9532 to 0.9570 , from 0.9528 to 0.9555 , and from 0.9527 to 0.9551 - that is, not more than 0.5 per cent. The mean is 0.95451 .

In further experiments according to another method, Fr. Weber placed the coils mentioned above at a definite distance from each other, connected the one, "the inducing coil," with a very constant Daniell's cell and a simple ring of $168 \cdot 7$ millim. radius; the other, "the induced coil," with a multiplier, which was composed of a coil consisting of two equal conical halves placed close together, of $154 \cdot 2$ millim. inner radius and $172 \cdot 2$ external radius, between which was suspended a magnet 40 millim. long provided with a mirror. The simple ring of the inducing circuit lay between these coils. The deviation of the needle whilst a constant current passed through the inducing circle measured its intensity $J$, which was to be measured in absolute measure from the known dimensions of the ring; the deviation on breaking the inducing circuit after disconnecting the ring gave the intensity $i$ of the induced current. Since the induced electromotive force $e=\mathrm{JP}^{\prime}$ (where $\mathbf{P}$ is the mutual potential of the coils) may be calculated in absolute measure from the dimensions of the coil by putting the constant of induction equal to unity, we obtain the resistance of the induced circuit $r$ from the formula $r=\frac{e}{i}$. 
We have therefore for these determinations to ascertain the dimensions (1) of the wire ring, (2) of the inducing coil, (3) of the induced coil, (4) of the multiplier, (5) of the distance of the separate layers of the inducing and induced coils from each other, (6) the determination of the position of the magnetic poles, to which are to be added the manipulations required for the other methods, the adjustment of the needle in the centre, and of the coils of the multiplier and of the wire ring between them with reference to the meridian \&c. Since the intensities of the primary and secondary currents are measured by the deflection of the same magnet, the horizontal component of the earth's magnetism does not enter into the calculation. There are in this method many more sources of error to be taken into account than in the other methods, by which its accuracy may be seriously impaired.

Notwithstanding, Weber finds, by determining the resistance of the inductive circuit by this method, and comparing it with the Siemens standard which he employed, that at two different distances of the inducing and induced coils, and with two different intensities of the inducing current, a mean result of $0.9554(0.9589 \text { to } 0.9516)^{*}$.

This method has also been employed by Rowland $\dagger$, only that he employed a tangent-galvanometer to determine the intensity of the inducing current, but a special galvanometer to determine the induced current; it was necessary also to determine the ratio of the intensities of horizontal force at the position of the two pieces of apparatus. His three inductioncoils had a diameter of about 27.4 centim., and were wound on frames having a thick brass rim on each side. They could be combined in pairs, so that the one which served as inductor, through which a constant current flowed which could be interrupted, might be placed at four different distances from the others $(6.5$ up to 11.47 millim.). Very thin-covered copper wire (no. 22) was taken for the coils; so that the sources of error mentioned on p. 260, caused by displacement of the covering, the mutual compression of the coils, and expansion of the wire, become very prominent. Since the in-

* An indirect method employed by Fr. Weber to determine the resistance of a zigzag platinum wire placed in a calorimeter, from the heat produced by a current whose strength in absolute measure was determined by means of a tangent-galvanometer of known dimensions, can hardly be placed by the side of the more direct methods, on account of the difficulty of all calorimetric measurements. Nevertheless Fr. Weber finds by this method, by comparison with his Siemens standard, a resistance for the latter of 0.9560 , which again differs only 0.1 per cent. from the other determinations.

$\dagger$ Rowland, 'Silliman's Journal,' 1878 (3), pp. 325, 430. 
duction-coils are so close together, a small error in measuring their distance apart is of great influence-the more so since, from what has been said, it follows that the position of the separate coils could not be accurately controlled. That the distance of the coils apart at various points was measured to the $\frac{1}{20}$ millim., and that the mean distance apart is given to Io 10 millim., and the mean radius to the ${ }_{10} 000$ millim., can hardly be regarded, in view of the above-mentioned disturbing causes, as a guarantee of the actual exactitude of the results.

Since the length of the needle of the tangent-galvanometer $\left(2 \cdot 7\right.$ centim.) amounted to only $\frac{1}{18}$ of the diameter of the coil (50 centim.), the deviation from the law of tangents is imperceptible ; but, on the other hand, there is an important source of error in the fact that the needle turned upon a point. Rowland asserts that the needle, which was provided with a pointer playing over a circle of 20 centim. diameter, always took up its position accurately to 1 or 2 minutes ( 1 minute corresponds to only 0.03 millim. upon the circle). Since this does not at all agree with previous experience, an exact explanation how so great sensitiveness was obtained is indispensably necessary.

The needle of the galvanometer was 1.25 centim. long; the coils, which were brought up to it from the east and west, had an internal diameter of 3 centim. and an outer diameter of $5 \cdot 6$ centim. ; their inner end-surfaces were at a distance of 0.935565 centim. (thus given to $10 \frac{1}{1000}$ millim.) from the point on which the needle turned. Whetber the law of tangents is altogether applicable here must remain uncertain.

Since the intensity of the inducing and induced currents is measured at different places, Rowland introduces a further complication by determining the ratio of the horizontal component of the earth's magnetism at the position of the tangentcircle and of the galvanometer by surrounding the galvanometer with a larger coil, sending the same current through it and the tangent-circle, and comparing the deflections. The radius of the new circle (some 4 centim.) is again given to ${ }_{10}^{1} \delta_{0} 0$ millim.

The values of the different dimensions given to so many decimal places are probably not regarded by the author himself as accurate in the same degree, the last decimals (obtained by interpolation) being added for the sake of completeness. It is, however, always advisable to give exact account how far actual observation goes, since the mean values can never be accurate beyond the limit of possible observation, or else with the roughest measures it would be possible by repeating measurements to obtain any required degree of minuteness. 
Since, moreover, the sources of error mentioned above may undoubtedly cause considerable deviations, such a one-sided accuracy cannot be a guarantee for the exactitude of the final results *.

On account of the much larger number of determinations which this method requires, I consider it much less suitable for the final construction of a definite resistance than the fourth and first of Weber's methods, of which again I give the preference to the latter.

After a conductor $\mathrm{A}$ of known absolute resistance has been constructed, by the one method or the other, it has to be compared with the resistance $B$ of a mercury column of known dimensions in order to determine the length of such a column of 1 square millimetre section which possesses $1 \mathrm{ohm}$ resistance.

If we employ Weber's first method, we may introduce the mercury column $\mathrm{B}$ directly between the inductor and multiplier, and calculate the resistance of $B$ from the decrease of intensity; otherwise the resistances A and B may be compared by means of the differential galvanometer, or more conveniently by means of the Wheatstone's bridge, according to the accurate method already employed by W. Siemens in constructing copies of his mercury unit. Since we already know the length of mercury corresponding to $1 \mathrm{ohm}$ very nearly, we take care to arrange the resistance $B$ so as to be as nearly as possible equal to the resistance $A$.

It is highly important, after calibration of the measuringwire, to secure perfect contacts by using freshly-amalgamated thick copper connecting-pieces and cups full of pure mercury, the resistance of which is previously determined by including several in a circuit. Plug-contacts are not sufficiently accurate. Also the temperature must be carefully kept constant throughout at the right point, as was the case in the construction of Siemens's copies.

* If we employ in this and other methods a coil wound uniformly on a closed ring, a neutral solenoid, as inductor, but as induced coil a coil surrounding the former at one point, the conditions are so far simpler that the current due to opening or closing the current in the solenoid, only the linear dimensions of the solenoid, its interior and exterior diameters $d$ and $d_{1}$, the number of coils of the induction-coil and of the solenoid, or in the case of a solenoid of rectangular section the height $a$, are concerned (compare Roiti, Atti di Torino, vol. xvii., 30th April 1882). Nevertheless the employment of such a solenoid is attended with the great difficulty of winding the wire exactly upon it, and of measuring its dimensions with sufficient accuracy. The latter is the more difficult, since the section of the solenoid can only be taken relatively small, and the formula contains the expressions $\sqrt{d}-\sqrt{d_{1}}$ for a solenoid with circular section, $a \log \frac{d_{1}}{d}$ for a solenoid with rectangular section. 
A difficulty presents itself here, since induced currents aro produced in $A$ on opening and closing the current which flows through the combination of wires. We must therefore either employ a constant current of such feeble intensity that the heating of the circuit shall be imperceptible, or, according to the method of Kohlrausch*, who has also worked out the necessary calculations, we must employ an induction-apparatus which sends alternating currents through the circuit. A special source of error is found in making contact with the mercury column contained in an accurately-calibrated earefully cleaned glass tube. The ends of the tube terminate in glass vessels of diameter relatively large. The apparatus is best filled with mercury by pouring mereury into one of the vessels and inclining the apparatus towards the same side, then, after closing the openings, repeatedly exhausting the apparatus as completely as possible, gently warming the tube, then allowing pure air which has passed over phosphoric anhydride and wadding to enter, and finally, after exhausting again, allowing the mercury to flow into the tube by placing it in a horizontal position. If the electrodes are immersed in the vessels containing mercury, then the resistance of the mercury in the tube is increased by that of the mercury contained in the vessels up to the ends of the electrodes. This resistance may be calculated on the assumption that the vessels are infinitely large. It might perhaps be advantageous to determine it directly by compensating the resistance of a tube filled with mercury with two contact-vessels by a noarly equal resistance on Wheatstone's bridge, and then to cut the tube at one or more points and to introduce wider mercury vessels of suitable form, and then again determine the resistance. We might also introduce two tubes, of lengths $m$ and $n$ and of equal sec.. tion, between the same contact-vessels, and compare their resistances, and from the data so obtained calculate the resistance of the vessels.

In order to avoid this separate comparison of the resistance of the coil determined in absolute measure with a mercury column, Carey Foster $\dagger$ and Lippmann $\ddagger$ have almost simultaneously suggested (L.) or carried out (C. F.) the ingenious plan of employing Weber's fourth method, as adopted by the British Association, and Poggendorff's compensation-method

* Poggendorff's Annalen, 1871, vol. cxlii. p. 418.

$\dagger$ Carey Foster, 'Electrician,' 1881, vol. vii. p. 266 ; Beiblittte', vol. vi. p. 133.

† Lippmann, Comptes Rendus, 1881, rol. xciii. p. 713; Beibläter, vol, vi. p. 43.

Phil, Mag. S. 5. Vol. 14. No. 88. Oct. 1882. 
for determining electromotive force without having previously determined the resistance of the rotating coil.

Let the resistance $R$ to be investigated and a tangent compass be included in the circuit of a constant pile, e.g. a thermopile. Let the ends of the resistance be connected also by a second circuit, into which a delicate galvanometer and the coil rotating about a vertical axis are introduced during a particular phase of its motion by means of a commutator revolving with the coil. The velocity of rotation is so regulated, or the resistance in the branch containing the constant pile is so altered, while the velocity of rotation of the coil remains constant, that the galvanometer is not affected. Then, at the moment that the coil is introduced, the induced electromotive force in it is equal to the difference of potential at the ends of the conductor $\mathbf{R}$ caused by the thermopile. If the current-intensity in it, and also in the circuit of the thermopile and of the tangent-galvanometer, be equal to $\mathrm{J}$, then $\mathrm{E}=J \mathrm{~J}$. The factor of reduction of the tangent-galvanometer is to be cletermined from its dimensions and the horizontal component of the earth's magnetism at the place, and in the same way the electromotive force induced by the rotation of the inductor. In most cases we may assume the horizontal component of the earth's magnetism to be the same at both places, as in Weber's first method, which thus disappears from the calculation. In this method, therefore, we have the same determinations to make as in Weber's fourth method. The uncertainty of contact in the commutator is without influence, since when the galvanometer is not affected there is no current in the inductor-ciretit. If we adjust the commutator so that it makes contact with the coil at the time of maximum induction, just at the moment when it varies least, then, if the ratios are in other respects properly chosen, the influence of self-induction in the rotating coil is reduced to a minimum. A certain amount of care, however, is required in order to avoid thermo-electric disturbances, resulting from the heating of the points of contact; and the determination of the exact phase during which the commutator makes contact offers a certain amount of difficulty.

The results so far obtained by the different methods are, some of them, tolerably far apart, although the investigations have been carried out with great care, if not in all points with the most perfect experimental means which a final determination of the ohm demands. Thus the resistance of a mercury column 1 metre long of 1 square millim. section at $0^{\circ} \mathrm{C}$. was found by the first experiments of the British Association to 
be 0.9830 ; according to F. Kohlrausch it is 0.9717 ; according to F. Weber 0.9550 ; and according to Lord Rayleigh and Mrs. Sidgwick $0.9413\left(10^{\circ} \frac{\text { centim. }}{\text { sec. }}\right)$. The first ohm constructed by the British Association, according to F. Kohlransch is 1.0196; according to Rowland 0.9910, and according to Rayleigh and Schuster 0.9893 of the true ohm ( $\left.10^{9} \frac{\text { centim. }}{\text { sec. }}\right)$.

Hence at any rate it is indicated that the final determination of the ohm must not rest alone on experiments made only according to one method and carried out at one place. Further, the results of each separate method (as I lave already mentioned) offer security against possible constant errors only if they are obtained from entirely independent series of experiments, made with apparatus raried in all possible ways. Since investigations are already in progress in different places, with excellent apparatus and according to different methods, we may shortly expect to be in a position to compare together the data which they yield, and so to attain as reliable a final result as possible.

In such important and permanent determinations as those of the electrical units, a delay of a few months is of no importance whatever in comparison with the reliability of the result to be obtained. Any introduction of the ohm as given by detached series of observations and distribution of copies for practical use would therefore be prematrre and without authority.

The Commission appointed for the determination of the electrical units does not complete its task by simply determining the ohm. It is further necessary to construct at lenst one of the two remaining units, e.g. the volt. Since it is at present impossible to construct this in a form capable of reproduction, it will be necessary first to compare electromotive forces with those of a constant element whose electromotive force is known in volts. We are therefore in the same case as if, wishing to measure a length in metres, we were obliged to employ a yard measure whose ratio to the metre was known. So much the more thoroughly are we obliged to investigate the electromotive forces of the galvanic cells to be employed as intermediate measures, and their dependence upon external conditions. The cell invented by Latimer Clark has indeed been shown to be capable of reconstruction of exactly the same electromotive force; at the same time it can only be employed for electrostatic measurements, since it becomes polarized when used to prodnce a current. Obser. 
vations with reference to Daniell's and other cells do not appear to me to lead to any certain conclusion.

Similar indirect methods have to be employed for measuring the intensity of a current in absolute measure; the factor of reduction of the tangent-galvanometer employed, for example, has to be determined. This problem has been considerably facilitated by the recent investigations of $\mathrm{F}$. Kohlrausch and Mascart on the electrochemical equivalent of silver, which, however, exhibit small deviations among themselves. There is here consequently a rich field for accurate investigation, for which we have already a series of valuable preliminary investigations. All these labours, however, can only give a final result sufficient for our present purpose when they are executed upon some common and well organized plan, and are carried out with the most perfect experimental means.

XXXI. Crystallographic Notes. By L. FLeTcher, M.A., of' the Mineral Department, British Museum; late Fellow of University College, Oxford*.

\section{[Plate VI.]}

\section{Un T'vins of Copper Pyrites.}

TTHE memoir of Haidinger $\dagger$ on the Crystallisations of 1 Copper Pyrites, published so long ago as the year 1822, was so exhaustive and withal so simple in its character that little seemed to be left to tempt the crystallographer to devote further study to this mineral; and in fact, with the exception of the papers of Sadebeck, whose early death all interested in the progress of mineralogy must so deeply deplore, and the confirmatory data in Groth's Catalogue of the Collection of the University of Strassburg, we have still no other information at our disposal. A study of these memoirs and of the various text-books of mineralogy leaves upon one's mind such a feeling of doubt as to the true statement of one of the laws of twin-growth, and that (as will be explained later) a law almost, if not quite, unique in the domain of crystallography, that, at the suggestion of Professor Maskelyne, the collection of copper pyrites in this Museum has been examined with a view to a possible settlement of the difficulty.

To get a clear idea of the present position, it is necessary to trace the history of this particular law from its first statement down to the present time.

In the above memoir of 1822 , the twin-growths of copper

* Read before the Crystallological Society, June 3, 1882.

+ Memoirs of the Wernerian Society, vol. iv. p. 1, 1822. 